\title{
Integrated Health Care System: Meeting Global I Health Care Needs in the 21st Century
}

\author{
Nat Quansah
}

\begin{abstract}
The Integrated Health Care System is presented as an appropriate system capable of helping meet global health care needs in a satisfactory manner. It is a system that consciously targets and harnesses peoples' links with biodiversity for health reasons. It is an integrative and positive tool blending health, economic, and biological and cultural diversity conservation needs of people and their area, all at the same time.
\end{abstract}

Results from a study of health care conducted from 1993 through 1997 at the 'Clinique de Manongarivo', a pilot rural health center in northwest Madagascar practicing the Integrated Health Care System approach, are used as evidence of the viability of this system. In conclusion a call is made for re-thinking and re-acting of health policies to implement the system in other countries.

\section{Introduction}

"HEALTH FOR ALL BY THE YEAR 2000". A laudable goal set by The World Health Organization (WHO) which unfortunately never became a reality. But we all aspire for GOOD HEALTH. This aspiration holds true for the individual, the family, the community, the country and the world as a whole. But why are we not achieving GOOD HEALTH FOR ALL? Many factors can be attributed to this failure, not least, the neglect of traditional medical systems. In many countries traditional medical systems are ignored as a result of the adoption of modern medical systems as the officially recognized medical systems.

The Integrated Health Care System (IHCS), a system that combines the resources (human and material) of different medical systems of an area in a complementary manner is the focus of this article. The IHCS has been studied from 1993 through 1997 in a pilot health care center in Madagascar: 'Clinique de Manongarivo'. Results from this pilot study are used in the following discussion to demonstrate how this system facilitates not only health care needs but also economic as well as biological and cultural diversity conservation needs of a people and their living area.

\section{Heath Policies: Adopting Modern Medical Systems}

Health policies in most countries (especially in developing countries) have often favored the adoption and development of modern medical systems while tolerating, belittling, neglecting or banning the existing (traditional) medical systems. This scenario, I believe, is the result of misconceptions arising from ignorance on the part of health authorities and administrators, modern medical personnel, scientists, and policy makers of the roles of the various medical systems, especially the traditional medical system as a profession and traditional medical practitioners as professionals. The situation may also be motivated, at times, by personal economic gains on the part of some proponents of modern medical systems. Proponents may include policy makers, pharmaceutical product manufacturers as well as health authorities and adminis-

\section{Correspondence}

Nat Quansah, Independent Researcher / Consultant, Health \& Environmental Issues, MADAGASCAR, natquansah@hotmail.com

Ethnobotany Research \& Applications 3:067-072 (2005) 
trators fragrantly disregarding the real health care needs of the country.

\section{Effects of Policies Limiting Health Care to Modern Systems}

\section{Net Loss}

The policy of adopting modern medical systems as the 'official medical systems' used to meet the health needs in most countries, has resulted in a net loss rather than a gain both health-wise and economically. Countries spend more on health but are unable to meet the health needs of the majority of their people.

\section{Non-Availability of Services}

The cost of adopting modern medical systems, economically, is beyond the reach of most countries (especially the developing ones). Services provided through these systems only manage to reach the few people who can afford them. The majority of people cannot afford the services provided by the system due to high costs of medicines (pharmaceutical products) as well as treatments. We forget, though that non-affordability leads to non-accessibility and results in non-availability. Any system that provides a service that is not affordable and therefore not accessible and not available can never claim to be effective and efficient. An effective and an efficient system must be able to deliver at most (if not at all) times in all circumstances and at all levels.

\section{Loss of Human Resources}

The loss is also seen in the areas of human resources availability and development. Replacing traditional medical system wholesale with modern medical system has meant doing away with capable health care providers, traditional medical practitioners. We neglect such a massive work force and turn to lament the lack of human resources in our health services. This is especially so when most rural areas hardly encounter modern medical personnel, whereas traditional medical practitioners are always present in these areas.

Similarly, as the majority of people cannot afford the services provided through the modern medical system, they risk having their diseases becoming chronic and/or dying from complications. The inability of these people to work at maximum capacity has adverse effects on human resource development in the productive sectors of the countries' economy. This loss is felt not only in the family life of individuals concerned but also in the society.

\section{Loss of Cultural and Biological Diversity}

Traditional medical system (especially, its aspect of using biodiversity - example, medicinal plants) is embedded in the traditions and cultures of most countries. Replacing this with the modern medical systems' use of pharmaceutical products shows the lack of respect for these cultures. This eventually results in the loss of this aspect of the culture as people end up turning their backs on and forgetting the knowledge and practices that go with the use of the plants. This is a loss in cultural diversity. The loss of cultural diversity, I believe, is one of the major contributing factors to the continued loss of biodiversity in many countries. This is because the links between people and biodiversity in their areas get severed. And when the links between people and other biodiversity in their areas have been severed it becomes difficult (if not impossible) for such a people to take up their responsibilities to help save biodiversity. Certain elements of cultural and biological diversities are inseparable hence the loss of one leads to the loss of the other.

Traditional medical systems (especially in Africa) have been belittled, tagged 'primitive', equated to the practice of witchcraft, often discouraged and banned. But as much as this system of ours may not be perfect, it certainly has strengths which if harnessed and properly managed will go a long way to help alleviate some of the hardships that many countries face, in terms of health care provision.

I believe we would do better if we were to adapt the modern medical system to local situations and make it complement traditional medical systems rather than adopting the modern medical system to replace traditional medical systems. The lack of respect for and disinterest in locally available resources reflect this tendency to adopt rather than to adapt. And this has resulted in many non-sustainable development programs in many countries.

\section{What Needs to be Done}

The foregoing does not mean that no progress has been made with regards to meeting health care needs globally. Advances have been made but there still remains a great deal more to be done before we can honestly say that we've been successful with this policy of adopting the modern medical system as official medical system. Adopting and developing modern medical system in many countries have not yielded the results that were envisaged. And if health policies remain as they are now, biased against traditional medical system but favoring the adoption and development of the modern medical system, then, attaining health for all will forever remain rhetoric and never a reality.

If the health strategy is not achieving its goal, then new and appropriate way(s) that will enable the health goal to be attained must be sought for. To be able to meet the health care needs of their people in a more effective and 
efficient manner would require that countries re-think and re-act their health policies and activities and come up with viable strategies founded on policies that reflect the realities of respective countries. It is in this context that I propose the Integrated Health Care System.

\section{The Integrated Health Care System (IHCS)}

The IHCS is a system that consciously targets and harnesses peoples' links with biodiversity (knowledge of and dependence on biodiversity, especially medicinal plants) for health reasons as a positive tool to arrive at meeting the health, economic as well as the biological and cultural diversity conservation needs of the people and their areas all at the same time.

\section{Philosophy of the IHCS}

The principles of conservation and that of the relay team together form the cornerstone of the IHCS. Let me briefly explain this.

Conservation: This is the management of the human use of the biosphere (the part of the earth's surface and atmosphere inhabited by living things) so that it may yield the greatest sustainable benefit to current generations while maintaining its potential to meet the needs and aspirations of future generations. At the heart of conservation is 'sustainable use'. This means using a resource in a way that it meets the needs and aspirations of the user without compromising the ability of the resource to meet the needs and aspirations of other users. Both present and future generations of users are taken into account by conservation.

Relay Team: This entails a group of individuals with different levels of competence coming together as one to compete for a common prize. Each individual carries out a specific task. For example: in a relay race, each member covers a specified portion of the total distance to be covered by the whole team. To be successful, team members have to work hard together at perfecting their abilities to pass on the baton. The members are always guided by the spirit of the relay team: respect for each other as a person and their capabilities, total confidence and trust in the other's ability to do his/her best for the good of the team. Team members' differences are harnessed and managed with their diversities to make sure that the team succeeds in attaining its goal. Each relay team member is independent but at the same time dependent.

As we can see, conservation emphasizes on the sustainable use of the diverse resources at ones' disposal while the success of the relay team depends on the ability to harness and manage individual differences and diversities to function in a complementary manner.
The principle of the IHCS is, thus, harness and manage the differences and the diversity of resources (material and human) available and use these in a complementary and sustainable manner.

The diverse local resources (material and human) must first be harnessed and used to the best of ones abilities before calling on external resources to come in to complement these. Learning to be independent and at the same time dependent is the key here. Local resources are not to be 'blindly replaced with so called alternatives'. External resources must come in to complement local competence as well as material resources. Trying to replace local resources rather than complementing them is not sustainable and tends to 'turn people (countries) into beggars' to the detriment of all.

\section{Resources (personnel and materials)}

Under the IHCS, resources (personnel and material) of the different medical systems of an area (traditional and modern medical systems) are combined with the traditional medical practitioner (TMP) and the modern medical practitioner (MMP) working together, complementing each other and using local biodiversity (medicinal plants etc..) and modern medicines (pharmaceutical products etc..) for the prevention and/or treatment of diseases. These (the TMP \& the MMP) then join forces with other specialists - botanists, chemists, ethnobotanists, pharmacologists, pharmacists, etc...- to complete the multi-disciplined team required to carry out the various activities necessary for the attainment of the IHCS goal.

\section{An example of a Health Center functioning on the basis of the IHCS}

Results from 1993 till end 1997 obtained at the 'Clinique de Manongarivo', a pilot health care center functioning on the principle of the IHCS in northwest Madagascar, form the basis of this part of my presentation. This is meant to demonstrate the validity and practicability of the IHCS.

Established in 1993, the 'Clinique de Manongarivo' in Ambodisakoana Village functioned with a multi-disciplined team divided into a Field Team (made up of traditional medical practitioner, modern medical doctors, ethnobotanists and local helpers) and a Laboratory Team (comprised mainly of chemists and pharmacologists). The team was charged to provide primary health care to the people of the Manongarivo area and arrive at the conservation of local biological and cultural diversity at the same time, as well as help the Clinic to attain self-sustaining status by the end of 1999. A combination of unfortunate circumstances resulting in the lack of funds, however, led to the suspension of the Clinics' activities at the end of 1997. This meant that the Clinics' self-sustaining status could not be met as planned. This unfinished task is to be pur- 
sued vigorously when the activities of the Clinic, planned to resume before the end of 2001, begin.

\section{Method of Working}

The team worked in a relay fashion with each other and with the local community.

\section{The Field Team}

The medical doctor and the traditional medical practitioner together received patients during consultations and examinations at the Clinic. They exchanged views and diagnoses after examination and then decided on the appropriate treatment. All information concerning the patient (clinical examination, investigation and treatment...) were noted in individual medical records (2 copies; one of which was kept at the Clinic, the other sent to the Laboratory Team). This allowed for easy follow-up of treatments and disease evolution of each patient. The follow-up was done either at the Clinic with the patient returning to the Clinic or at patients' home with doctor and traditional medical practitioner visiting.

Initially, priority was given to choosing traditional medicine (medicinal plants) with the traditional medical practitioner carrying out the prescription. The medical doctor prescribed a pharmaceutical product only when there was no local remedy for the case in hand. This was to enable the Clinic be able to maximize benefits from the use of local resources before bringing in external resources to complement the local ones.

Medicinal plants prescribed were prepared in the presence of the patient. This was meant to teach patients (and/or the person accompanying them) how to prepare the treatment correctly should the need arise and help limit and finally remove the dangers associated with the misuse of medicinal plants. The issue of 'not knowing the right dose to use' is often used to discredit traditional medicine, albeit incorrectly. The teaching aspect of the functioning of the Clinic was meant to help correct this. The patient (and/ or accompanying person) was taught to understand that the quantity of the medicine, the frequency as well as the duration of taking it is important in every treatment. They were therefore encouraged to apply this always, irrespective of the treatment type. Using the correct dose would mean avoiding waste and eliminating the dangers through the use of too much or too little material. This then results in the sustainable use of the resource.

Patients whose treatments required the prescription of modern medicine paid for the cost of purchasing these medicines from the Clinic's pharmacy.

\section{Relay}

Data from the Clinic, as per the copied medical records (empirical information on medicinal plants provided by the traditional medical practitioner and used in the Clinic, the medical result noted during treatment and follow-up) were sent to the Laboratory Team for further investigation.

\section{The Laboratory Team}

The laboratory team sought to authenticate the empirical use of the medicinal plants, standardize the known effective remedies and provide information that enabled the field team and the community to arrive at maximizing the benefits to be gained by the sustainable use of local medicinal plants.

To this effect, research was carried to select the most effective medicinal plant(s) for a particular disease and the most effective part of the plant to be used. Similarly, investigation on secondary effects, toxicity and undesired effects as well as the improvement of the method of preparation of the medicinal plant(s) were undertaken. All laboratory results were returned through the field team to the community to help optimize efficiency and conservation. Plants recommended for use in the Clinic and by the community had to be: plants already known and used by the community and/or the traditional medical practitioner, effective, less toxic and with wide distribution.

\section{Results}

Activities carried out by Clinic resulted in the practice of three treatment types. Diseases were treated by:

- medicinal plant(s) only

- modern medicine(s) only

- the combination of medicinal plant(s) and modern medicine(s).

Of the 36 different diseases encountered at the Clinic, $77.78 \%$ were strictly treated effectively by local medicinal plants; $5.56 \%$ by pharmaceutical products while the other $16.66 \%$ were treated using a combination of medicinal plants and pharmaceutical products.

Diseases or symptoms treated
with medicinal plants only:
Abscess
Asthma
Athletes' foot
Burns
Constipation
Cough
Diarrhea
Dysentery
Dysmenorrhea
Dysuria




Fatigue (general)
Fever
Gonorrhea
Headache
Herpes
Hypertension
Indigestion
Influenza
Intestinal worms
Jaundice
Measles
Miscarriage
Motion sickness
Scabies
Stomachache
Stomach ulcer
Toothache
Wounds

Diseases treated with modern medicines only: Acute bronchopneumonia Typhus fever syndrome

\section{Diseases treated by combining medicinal plants and modern medicines: \\ Chronic bronchopneumonia \\ Cough (with acute chest infection) \\ Genital ulcers \\ Malaria \\ Syphilis \\ Wounds (with complications)}

\section{Sustainable use of medicinal plants}

The complementary functioning of the personnel of the IHCS Clinic during this period saw the selection of appropriate plants and plant parts for the effective treatment of various cases encountered in the area. For example, laboratory investigations led to the use of the leaves of a Burasaia sp. for the treatment of fever in the Clinic instead of the earlier use of the roots of this plant by the traditional medical practitioner and the community. Also, the oil extracted from the fruits of a Mauloutchia sp. was used to treat toothache in place of using the bark.

A medicinal plant's garden was established on the premises of the Clinic. This garden has 30 different species of medicinal plants growing in it. The plants from the garden were used in the Clinic in order to take undue pressure off some of the plants of the area. This is in line with the sustainable resource use principle of the IHCS.

\section{The Cost-Effectiveness of the IHCS}

The functioning of the 'Clinique de Manongarivo' based on the principle of the IHCS brings into light what I consider to be the five pillars of health care service: affordability, accessibility, availability, effectiveness and efficiency all at the same time.

With its multi-disciplined team and combined resources, the IHCS becomes a system that minimizes waste while maximizing benefits. The provision of health care services, under the IHCS is economically affordable, accessible, available, effective, efficient and culturally acceptable.

\section{Economic as well as biological and cultural diversity conservation gains}

By reinforcing the use of local medicinal plants, which is often free (at worst 5 to 10 times less expensive than the pharmaceutical product) the IHCS Clinic enabled patients to cut down on their health care costs. People saved money by using medicinal plants for the diseases that these plants could take care of rather than relying solely on pharmaceutical products for all diseases. For example: people saved around US\$5 for using ginger to take care of their motion (travel) sickness; US\$7 for using a Croton sp. in treating diarrhea; up to US\$20 in using an Erythroxylum $\mathrm{sp}$. to take care of their asthma and around US\$35 for using a Mauloutchia sp. for herpes.

This made it possible for them to afford the cost of pharmaceutical products needed for the other disease(s) that had no effective local biodiversity as remedy. The money saved was also used to help meet other family needs, such as paying for the cost of a child's education. Note that pharmaceutical products are usually 3 to 4 times more expensive in rural areas than in the urban centers and often more difficult to find.

The use of medicinal plants (biodiversity) is a cultural practice. Any system, therefore that strengthens peoples links with biodiversity easily becomes accepted by people as these are able to identify with it. Hence, the acceptance of the IHCS in the Manongarivo area. As the IHCS reinforces the life-saving value of plants through their uses for health reasons, it makes it easier to evoke peoples' willingness to take up their responsibilities to help conserve local biodiversity. They depend on local biodiversity for survival and so felt obliged to save biodiversity. Arriving at the continued and sustainable use of medicinal plants meant saving that aspect of the culture from disappearing.

The pilot IHCS Clinic in Ambodisakoana Village resulted not only in making appropriate health care services available to the people, it also brought in economic, biological and cultural diversity conservation gains.

\section{Preventive Activities}

'Prevention is better than cure' so goes the adage. As a result of this, vaccination, family planning, health education as well as discussions on sustainable resource use and management were other activities carried out at the 
Clinic in order to help ensure the general well-being of the people and the durability of the activities carried out through the IHCS.

Let me terminate my presentation with the following: I came across a pamphlet of the Pharmaceutical Manufacturers Association of Canada which read: 'of all known diseases, only a third have been successfully treated'. I believe this 'successfully treated' applies to working through the modern medical system and using pharmaceutical products. Assuming, a Traditional Medicine Manufacturers Association also existed and this also had a pamphlet that read: ' of all known diseases, only a third have been successfully treated' and this was to refer to working through the traditional medical system with its use of traditional medicines.

The above statements clearly show that each of the systems, separately, would be failing to meet global health care needs in a satisfactory manner. What happens to the remaining two-thirds in each case? If these two systems (ancient and modern) were to work together to complement each other, the results, I'm sure would be better than with each system on its own.

It is in this context of achieving a better result that I propose the IHCS approach to global health care provision. This system harnesses differences and enables people with diverse specialties to work to complement each other. The IHCS enables bridges to be built to link seemingly non-linkable domains unlike other systems that tend to erect fences so as to work in isolation, opposing and oftentimes seeking to destroy each other to the detriment of all.

Finding successful way(s) to meet global health needs more effectively and efficiently, would require a re-think and a react of health policies and activities based on honesty. I call for this re-think and re-act so as to allow health care providers willing to adapt and implement the IHCS approach in various countries to be able to do so.

Yes, we missed the target of the Year 2000 for 'Health for all'. But for how long are we going to continue to miss and deny the human race and our environment a good health for all? Adapting the IHCS will not only help solve the health care problems of countries it will also help meet the economic as well as biological and cultural diversity conservation needs. The time to be real and act is now.

Let us turn our words into works.

\section{Acknowledgements}

The moral, financial and material supports of diverse individuals and institutions made it possible for the dream of an Integrated Health Care System program to become a reality in the Manongarivo area. I'm grateful to all. The greatest appreciation, however, go to the people of the Manongarivo area whose dedication to and acceptance of the program and its members enabled the successful pilot stage to be attained. 